\title{
VLBA DETERMINATION OF THE DISTANCE TO NEARBY STAR-FORMING REGIONS. V. DYNAMICAL MASS, DISTANCE, AND RADIO STRUCTURE OF V773 Tau A
}

\author{
Rosa M. Torres ${ }^{1}$, Laurent Loinard ${ }^{2,5}$, Amy J. Mioduszewski ${ }^{3}$, Andrew F. Boden ${ }^{4}$, Ramiro Franco-Hernández ${ }^{1,6}$, \\ WOUTER H. T. Vlemmings ${ }^{1,7}$, AND LuIS F. RodRÍGUEZ ${ }^{5,8}$ \\ ${ }^{1}$ Argelander-Institut für Astronomie, Universität Bonn, Auf dem Hügel 71, D-53121 Bonn, Germany; rtorres@ astro.uni-bonn.de \\ ${ }^{2}$ Max-Planck-Institut für Radioastronomie, Auf dem Hügel 69, D-53121 Bonn, Germany \\ ${ }^{3}$ Dominici Science Operations Center, National Radio Astronomy Observatory, 1003 Lopezville Road, Socorro, NM 87801, USA \\ ${ }^{4}$ Division of Physics, Math, and Astronomy, California Institute of Technology, 1200 East California Boulevard, Pasadena, CA 91125, USA \\ ${ }^{5}$ Centro de Radiostronomía y Astrofísica, Universidad Nacional Autónoma de México, Apartado Postal 72-3 (Xangari), 58089 Morelia, Michoacán, Mexico \\ Received 2011 March 18; accepted 2011 November 30; published 2012 February 10
}

\begin{abstract}
We present multi-epoch Very Long Baseline Array (VLBA) observations of V773 Tau A, the 51 day binary subsystem in the multiple young stellar system V773 Tau. Combined with previous interferometric and radial velocity measurements, these new data enable us to improve the characterization of the physical orbit of the A subsystem. In particular, we infer updated dynamical masses for the primary and the secondary components of $1.55 \pm 0.11 M_{\odot}$ and $1.293 \pm 0.068 M_{\odot}$, respectively, and an updated orbital parallax distance to the system of $135.7 \pm 3.2 \mathrm{pc}$, all consistent with previous estimates. Using the improved orbit, we can calculate the absolute coordinates of the barycenter of V773 Tau A at each epoch of our VLBA observations, and fit for its trigonometric parallax and proper motion. This provides a direct measurement of the distance to the system almost entirely independent of the orbit modeling. The best fit yields a distance of $129.9 \pm 3.2 \mathrm{pc}$, in good agreement (i.e., within $1 \sigma$ ) with the distance estimate based on the orbital fit. Taking the mean value of the orbital and trigonometric parallaxes, we conclude that V773 Tau is located at $d=132.8 \pm 2.3 \mathrm{pc}$. The accuracy of this determination is nearly one order of magnitude better than that of previous estimates. In projection, V773 Tau and two other young stars (Hubble 4 and HDE 283572) recently observed with the VLBA are located toward the dark cloud Lynds 1495, in the central region of Taurus. These three stars appear to have similar trigonometric parallaxes, radial velocities, and proper motions, and we argue that the weighted mean and dispersion of their distances $(d=131.4 \mathrm{pc}$ and $\sigma_{d}=2.4 \mathrm{pc}$ ) provide a good estimate of the distance to and depth of Lynds 1495 and its associated stellar population. The radio emission from the two sources in V773 Tau A is largely of gyrosynchrotron origin. Interestingly, both sources are observed to become typically five times brighter near periastron than near apastron (presumably because of increased flaring activity), and the separation between the radio sources near periastron appears to be systematically smaller than the separation between the stars. While this clearly indicates some interaction between the individual magnetospheres, the exact mechanisms at play are unclear because even at periastron the separation between the stars $\left(\sim 30 R_{*}\right)$ remain much larger than the radius of the magnetospheres around these low-mass young stars $\left(\sim 6 R_{*}\right)$.
\end{abstract}

Key words: astrometry - binaries: spectroscopic - radio continuum: stars - stars: formation - stars: individual (V773 Tau) - stars: pre-main sequence

\section{INTRODUCTION}

In spite of significant progress in recent years, the formation and early evolution of stars are still not fully understood (e.g., Hillenbrand \& White 2004; Mathieu et al. 2007). One way to foster progress is to measure as accurately as possible the intrinsic characteristics of individual young stars (luminosity, effective temperature, mass, disk properties, etc.), and compare them with the predictions of detailed theoretical models. Young binary systems are particularly interesting in this respect because tracking their orbital motions provides a direct means to estimate their dynamical mass. In particular, if astrometric and spectroscopic data are combined, the physical orbit and the individual masses of the system members can be determined.

A recurrent obstacle to the accurate determination of the intrinsic properties of young stars has been fairly large

\footnotetext{
6 Also at Departamento de Astronomía, Universidad de Chile, Casilla 36-D, Santiago, Chile.

7 Also at Onsala Space Observatory, SE-439 92 Onsala, Sweden.

8 Also at Astronomy Department, Faculty of Science, King Abdulaziz University, P.O. Box 80203, Jeddah 21589, Saudi Arabia.
}

uncertainties (typically 20\%-50\%) in distance estimates to even the nearest star-forming regions. Significant progress has been possible in recent years, thanks to direct trigonometric parallax measurements obtained using Very Long Baseline Interferometry (VLBI) multi-epoch observations. Such results have been reported, in particular, in the previous papers in this series (Loinard et al. 2005, 2007, 2008; Torres et al. 2007, 2009; Dzib et al. 2010, 2011). They provide uncertainties of a few percent or better that typically surpass the accuracy of previous determinations by one order of magnitude.

V773 Tau (HD 283447, HBC 367) is a young stellar system located toward the dark cloud Lynds 1495 in Taurus. V773 Tau was first identified as a T Tauri star by Rydgren et al. (1976) and was established as a visual double (components designated A and B) with an apparent separation of roughly 150 mas in high angular resolution studies independently by Ghez et al. (1993) and Leinert et al. (1993). Roughly contemporaneously, Martin et al. (1994) and Welty (1995) established the brighter (A) visual component as a short-period (51 days) doublelined spectroscopic binary. Duchêne et al. (2003; hereafter D2003) and Woitas (2003) independently identified a third 
visual component (designated C; note that D2003 use an alternate component notation) in the system, indicating that V773 Tau is (at least) a quadruple system. ${ }^{9}$

V773 Tau A has long been known to be a strong radio source (Kutner et al. 1986). ${ }^{10}$ Indeed, it was the strongest source in the $5 \mathrm{GHz}$ Very Large Array (VLA) survey of weak line $\mathrm{T}$ Tauri stars (WTTS) in the Taurus-Auriga molecular cloud complex by O'Neal et al. (1990). From detailed multi-frequency observations, Feigelson et al. (1994) concluded that the radiation was most likely of non-thermal origin. This was confirmed by Phillips et al. (1996, hereafter P1996) who obtained VLBI observations, and resolved the radio emission into a clear double source, most likely corresponding to the two components of the spectroscopic binary. More recently, Massi et al. (2002, 2006) showed that the radio emission exhibits periodic variations with a period corresponding to the 51 day orbital period of the spectroscopic binary. This variability is due to an increase in the flaring activity near periastron and might reflect interactions between extended magnetic structures associated with the two stars when they get close to one another. Finally, Boden et al. (2007, hereafter B2007) and Massi et al. (2008) also resolved the radio emission from V773 Tau A into two components, which they associate with the two stars in the spectroscopic binary.

The dynamics in V773 Tau has been studied by a number of authors. Relative orbital motion among the A, B, and $\mathrm{C}$ components has been monitored by D2003, B2007, and Boden et al. (2012, a companion paper to this one, hereafter B2012). Following Welty (1995) and P1996, B2007 used radio and nearIR interferometry and spectroscopic radial velocity (RV) data sets to estimate the A-subsystem physical (three-dimensional) orbit. They obtain dynamical mass estimates of 1.54 and $1.33 M_{\odot}$ for the $\mathrm{Aa}$ and $\mathrm{Ab}$ components, respectively. Further, B2007 estimated the A-subsystem distance by means of "orbital parallax" (comparing the angular and physical orbit size), yielding $136.2 \pm 3.7 \mathrm{pc}$. There is also a direct trigonometric parallax measurement based on multi-epoch VLBI observations for this source (Lestrade et al. 1999). This VLBI-based distance measurement $\left(d=148.4_{-5.3}^{+5.7} \mathrm{pc}\right)$ is roughly consistent (at the $2 \sigma-3 \sigma$ level) with the value obtained from the A-subsystem orbit modeling.

In this paper, we present new VLBI observations of V773 Tau A which resolve the A subsystem and yield additional insights on its structure. These data are described in Section 2, and modeled jointly with earlier observations from B2007 in Section 3 to update the A-subsystem physical orbit and resulting physical parameters (component dynamical masses, orbital parallax). Then the updated A orbit model is used in conjunction with the VLBA global astrometry to compute a new trigonometric parallax to V773 Tau A. The relevance of these new results for the distance to the dark cloud Lynds 1495 is discussed in Section 3.3. Several interesting features of the V773 Tau A radio emission are apparent in these new observations;

\footnotetext{
9 It should be noted that multiple nomenclatures have been used to describe the components of V773 Tau. D2003 designate the spectroscopic binary V773 Tau A/B, and the optical and infrared companions V773 Tau C and V773 Tau D, respectively. Alternately, White \& Ghez (2001), Woitas (2003), and Massi et al. (2008), among others, designate the spectroscopic binary as V773 Tau A (containing stars $\mathrm{Aa}$ and $\mathrm{Ab}$ ), and the two companions as $\mathrm{B}$ and $\mathrm{C}$, respectively. A third nomenclature is used in the Double Star Catalog of Mason et al. (2001), which designates the spectroscopic binary Aa, containing stars Aa1 and Aa2. In this work, we will follow the notation established by White $\&$ Ghez (2001), reflecting the hierarchical nature of the V773 Tau architecture.

10 The two companions V773 Tau B and C, on the other hand, are not detected at radio wavelengths at the level of sensitivity of existing observations.
}

Table 1

Project Code and Date for Each Observation

\begin{tabular}{|c|c|c|c|}
\hline Epoch & Project Code & $\begin{array}{c}\text { Mean UT Date } \\
\text { (yyyy.mm.dd hh:mm) }\end{array}$ & Julian Day \\
\hline 01 & BM 198 A & $2004.03 .1120: 12$ & 2453076.3417 \\
\hline 02 & BM 198 B & $2004.03 .12 \quad 20: 12$ & 2453077.3417 \\
\hline 03 & BM $198 \mathrm{C}$ & $2004.03 .1320: 12$ & 2453078.3417 \\
\hline 04 & BM 198 D & 2004.03.14 20:12 & 2453079.3417 \\
\hline 05 & BM $198 \mathrm{E}$ & 2004.03.15 20:11 & 2453080.3414 \\
\hline 06 & BM $198 \mathrm{~F}$ & 2004.03.16 20:11 & 2453081.3414 \\
\hline 07 & BM $198 \mathrm{G}$ & 2004.03.17 20:11 & 2453082.3414 \\
\hline 08 & BL 128 AA & 2005.09.08 12:01 & 2453622.0013 \\
\hline 09 & $\mathrm{BL} 128 \mathrm{AB}$ & $2005.11 .1507: 31$ & 2453689.8136 \\
\hline 10 & BL $128 \mathrm{AC}$ & 2006.01.21 03:11 & 2453756.6327 \\
\hline 11 & BL $128 \mathrm{AD}$ & 2006.04.01 22:31 & 2453827.4386 \\
\hline 12 & BL $128 \mathrm{AE}$ & 2006.06.12 17:48 & 2453899.2423 \\
\hline 13 & BL $128 \mathrm{AF}$ & 2006.09.05 12:14 & 2453984.0102 \\
\hline 14 & BL 146 B & 2007.08.23 13:06 & 2454336.0461 \\
\hline 15 & BL $146 \mathrm{C}$ & $2007.08 .29 \quad 12: 42$ & 2454342.0295 \\
\hline 16 & BL $146 \mathrm{D}$ & 2007.09.05 12:15 & 2454349.0106 \\
\hline 17 & BL $146 \mathrm{E}$ & 2007.09.11 11:51 & 2454354.9943 \\
\hline 18 & BL $146 \mathrm{~F}$ & 2007.09.16 11:32 & 2454359.9806 \\
\hline 19 & BL $146 \mathrm{G}$ & 2007.09.21 11:12 & 2454364.9669 \\
\hline 20 & BL $146 \mathrm{H}$ & $2007.09 .27 \quad 10: 48$ & 2454370.9504 \\
\hline 21 & BL $146 \mathrm{I}$ & $2007.10 .03 \quad 10: 25$ & 2454376.9342 \\
\hline 22 & BL $146 \mathrm{~J}$ & 2007.10.09 10:01 & 2454382.9177 \\
\hline 23 & BL $146 \mathrm{~K}$ & 2007.10.17 09:30 & 2454390.8960 \\
\hline 24 & BL $146 \mathrm{~L}$ & 2007.10.23 09:06 & 2454396.8794 \\
\hline 25 & BL $146 \mathrm{M}$ & 2007.10.27 08:50 & 2454400.8684 \\
\hline 26 & BL $146 \mathrm{~N}$ & 2007.11.17 07:28 & 2454421.8114 \\
\hline 27 & BM 306 & 2009.09.27 08:07 & 2455101.8386 \\
\hline
\end{tabular}

the insights that they yield on the magnetospheric physics of the A-subsystem components are discussed in Section 4.2.

\section{OBSERVATIONS AND DATA CALIBRATION}

In this paper, we will make use of a total of 27 continuum observations of V773 Tau A obtained with the VLBA at $\lambda=$ $3.6 \mathrm{~cm}$ (see Table 1 for details). These observations correspond to four different projects (labeled BM198, BL128A, BL146, and BM306) that we will now describe separately.

\section{1. $B M 198$}

This first series of observations is archival (PI: M. Massi) and corresponds to the data published initially by B2007. It consists of seven observations (epochs 1-7 in Table 1) obtained every day between 2004 March 11 and 17, when the system was near apastron. The phase center was at $\alpha_{\mathrm{J} 2000.0}=04^{\mathrm{h}} 14^{\mathrm{m}} 12^{\mathrm{s}} .9198$, $\delta_{\mathrm{J} 2000.0}=+28^{\circ} 12^{\prime} 12^{\prime \prime}$. 199. Six hours of telescope time were allocated to each epoch, and each observation consisted of series of cycles with four minutes spent on source, and two minutes spent on the main phase-referencing quasar J0403+2600, located 3.31 away. Every 30 minutes, the secondary calibrator J0408+3032 was also observed. The data were collected with the VLBA plus Effelsberg, but for consistency with the rest of the data used in this paper (which were all VLBA only), the Effelsberg antenna was not included during this data reduction.

\section{2. $B L 128 A$}

The second set of data consists of six observations obtained every two to three months between 2005 September and 2006 September (epochs 8-13 in Table 1), and was initially designed to follow the parallactic path of the source. 


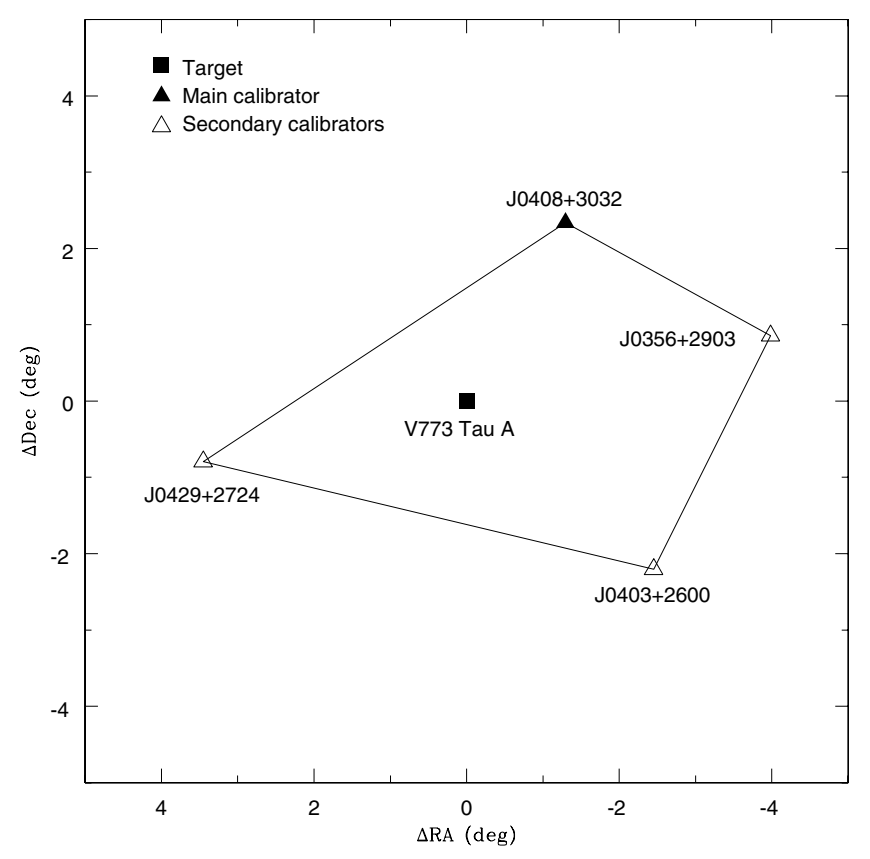

Figure 1. Relative position of the astronomical target, the main calibrator (J0408+3032), and the secondary calibrators (J0403+2600, J0429+2724, and J0356+2903) for projects BL128A, BL146, and BM306.

The phase center was at $\alpha_{\mathrm{J} 2000.0}=04^{\mathrm{h}} 14^{\mathrm{m}} 12^{\mathrm{s}} .9195, \delta_{\mathrm{J} 2000.0}=$ $+28^{\circ} 12^{\prime} 12^{\prime \prime}$.291. Two hours of telescope time were allocated to each epoch. Each observation consisted of series of cycles with two minutes spent on source, and one minute spent on the main phase-referencing quasar J0408+3032, located 2.67 away. Every 24 minutes, we also observed three secondary calibrators (J0403+2600, J0429+2724, and J0356+2903) which, together with $\mathrm{J} 0408+3032$, form a lozenge around the astronomical source (Figure 1). All four calibrators are very compact extragalactic sources whose absolute positions are known to better than $0.58,0.40,0.41$, and 1.46 mas, respectively (taken from L. Petrov, solution 2008, unpublished, available at http://vlbi.gsfc.nasa.gov/).

\section{3. $B L 146$}

This third data set corresponds to 13 observations obtained roughly every 7 days between 2007 August 29 and November 17 (epochs 14-26 in Table 1). These observations span a total of 82 days (about 1.6 orbital periods of the system) and were designed to follow the orbital motion over a complete revolution. In this case, the phase center was at $\alpha_{\mathrm{J} 2000.0}=04^{\mathrm{h}} 14^{\mathrm{m}} 12^{\mathrm{s}} .9213$, $\delta_{\mathrm{J} 2000.0}=+28^{\circ} 12^{\prime} 12^{\prime \prime}$. 190 , and five hours of telescope time were allocated to each epoch. The observing strategy (with cycles of two minutes on source and one minute on the main calibrator) and the calibrators were the same as those used for project BL128A.

\subsection{BM306}

Finally, a single observation of V773 Tau A was obtained in 2009 September (epoch 27 in Table 1) when the source was near periastron. The data were collected using the High Sensitivity Array (HSA) which combines the VLBA with the phased VLA, and the Arecibo, Green Bank, and Effelsberg telescopes. However, for consistency with the other observations presented in this paper, only the VLBA antennas were retained during the data calibration. The phase center was at $\alpha_{\mathrm{J} 2000.0}=$ $04^{\mathrm{h}} 14^{\mathrm{m}} 12^{\mathrm{s}} .9262, \delta_{\mathrm{J} 2000.0}=+28^{\circ} 12^{\prime} 12^{\prime \prime}$. 102 . Six hours of telescope time were allocated to the observation in this project, and the observing strategy was the same as for projects BL128A and BL146.

All four sets of data were edited and calibrated in a similar fashion using the Astronomical Image Processing System (AIPS; Greisen 2003). The basic data reduction followed the standard VLBA procedure for phase-referenced observations. For the projects BL128A, BL146, and BM306, the data processing included the multi-source calibration strategy described in detail in Torres et al. (2007) and Torres (2010; Sections 4.2-4.5). This was not applied to the data corresponding to the archival project BM198, which did not include the necessary secondary calibrators scans. Once calibrated, the visibilities were imaged with a pixel size of $50 \mu$ as applying weights intermediate between natural and uniform (ROBUST $=0$ in AIPS; Briggs 1995) to optimize the compromise between angular resolution and sensitivity.

Obtaining polarimetric information is important to characterize the radio emission mechanisms, but requires specific additional calibration scans (in the present case, observations of 3C84 at the beginning, the middle, and the end of each observing session) which were only obtained as part of project BL146 (epochs 14-26 in Table 1). These observations are used to determine and correct for several instrumental and atmospheric effects still corrupting the measured cross-correlations after the standard calibration procedure described above. Specifically, our polarization calibration consisted of two steps. First, we calibrated the cross-polarization delays using observations of the very strong calibrator $3 \mathrm{C} 84$ to remove differences in single and multiband delays for the right- and left-handed components. Second, we computed instrumental polarization corrections to calibrate the feed impurities (the so-called D-terms) using observations of $3 \mathrm{C} 84$ to determine the effective feed parameters for each antenna and IF. Finally, the data were imaged in Stokes parameter $V$ (circular polarization) with the same pixel size used for Stokes $I$ (50 $\mu \mathrm{as})$. An analysis of the issue of linear polarization will be deferred to a forthcoming paper.

One important aspect concerning astrometry must be mentioned here. The astrometric information derived from VLBI observations is contained in the complex visibility phases calibrated against the main calibrator used during the observations. As a consequence, it is very important (1) to use a main calibrator as compact and structureless as possible, and (2) to use the same calibrator for all observations. The last three sets of data used here (BL128A, BL146, and BM306) were obtained using the same main calibrator $(\mathrm{J} 0408+3032)$, which is indeed very compact and largely structureless (Figure 2(a)). Project BM198, however, was not originally intended as an astrometric experiment and used a different calibrator $(\mathrm{J} 0403+2600)$ which has a well-resolved core-jet structure (Figure 2(b)). This source was used as a secondary calibrator for the other three projects, so the source positions derived from project BM198 can be indirectly registered to $\mathrm{J} 0408+3032$. The poorer quality of the main calibrator and the indirect nature of the astrometry registration imply, however, that the astrometry derived from project BM198 is somewhat less accurate than that of the other three projects.

\section{ASTROMETRY}

The images of V773 Tau A obtained at all 27 epochs are shown in Figure 3. A well-resolved double-source morphology 

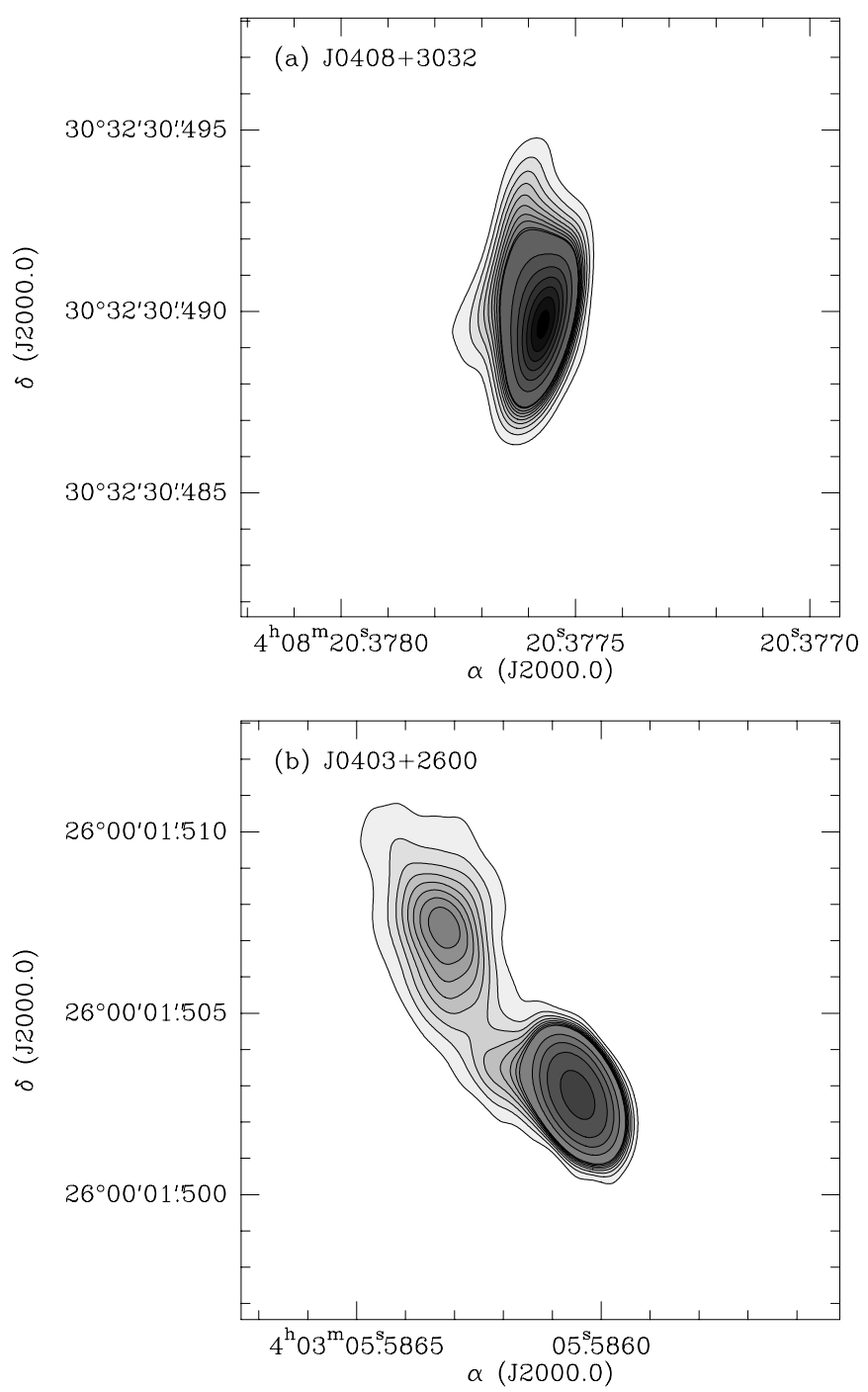

Figure 2. Images of the two main calibrators used in this paper. (a) $\mathrm{J} 0408+3032$. The noise level in the image is $\sigma=0.18 \mathrm{mJy}$, and the contours are at $5 \sigma-50 \sigma$ by $5 \sigma$ and at $0.01-0.07 \mathrm{Jy}$ by $0.01 \mathrm{Jy}$. (b) $\mathrm{J} 0403+260$. The noise level in the image is $\sigma=0.53 \mathrm{mJy}$, and the contours are at $5 \sigma-50 \sigma$ by $5 \sigma$ and at $0.05,0.1$, 0.2 , and $0.5 \mathrm{Jy}$.

(corresponding to the two stars in V773 Tau A) is detected at 20 of the 27 epochs, while a single source is seen in the remaining seven epochs. The source positions (Table 2) were determined using a two-dimensional Gaussian fitting procedure (task JMFIT in AIPS); when the source was double, both components were fitted simultaneously. JMFIT provides an estimate of the errors on the source position based on the expectation for ideal interferometer data. However, in typical VLBI observations where the main calibrator is located a few degrees from the astronomical target (such as those considered here), remaining systematic phase calibration errors normally dominate the error budget, so the errors provided by JMFIT underestimate the true uncertainties. We will come back to this point later.

\subsection{Relative Astrometry and Orbit Modeling}

In this section, we will concentrate on the 20 epochs when the source was double and analyze the relative position of the two sub-components. As established by P1996 and B2007, these double-source observations reflect the relative astrometry of the
V773 Tau A components, ${ }^{11}$ and as such can be used to assess and update the physical orbit obtained by B2007. The angular separations $(\Delta \alpha, \Delta \delta)$ between the primary and the secondary are given in Table 3 for the 20 epochs when the source is double. The uncertainties on $(\Delta \alpha, \Delta \delta)$ quoted in Table 3 are based on the errors delivered by JMFIT and are almost certainly underestimated (see above).

Figure 4 shows all available relative astrometry data on V773 Tau A (from P1996, B2007, and this work), along with several orbit models including that from B2007 (dashed line). Clearly, there is general agreement between the old and new VLBI relative astrometry on V773 Tau A and the a priori B2007 orbit model. A close inspection of the VLBI separations and the B2007 orbit model in Figure 4, however, shows an interesting trend in the VLBI astrometry. At most orbit phases the VLBI-derived separations and B2007 orbit model are in good agreement. But in most observations near periastron (secondary southwest of the primary) the VLBI separations appear systematically smaller (i.e., secondary nearer the primary) than predicted by the B2007 orbit. This suggests one of the two possibilities: there is a possible bias in the B2007 orbit solution or a possible bias in the VLBI-derived astrometry near periastron (where there is known enhancement in the radio flaring; Massi et al. 2002, 2006).

To investigate this issue we considered two different prescriptions for integrating the new VLBI data into the orbit modeling. In the first "VLBI + RV" model, we considered the possibility that the Keck Interferometer (KI) visibilities were the source of a possible orbit bias in B2007 and used only the (old and new) VLBI-derived relative astrometry and double-lined RV from B2007 to derive an orbit model. In the second "Full Hybrid" model, we used all available data (VLBI astrometry, KI visibilities, and RV), but assume that the VLBI separations near periastron were biased by the enhanced flaring activity (e.g., if the enhanced flares preferentially occur between the two stars), assigning these points lower weight (specifically a factor of $50 \%$ larger $1 \sigma$ error per axis for orbit phases within $10 \%$ of periastron). ${ }^{12}$ We find that both new orbit solutions are in excellent agreement with the original B2007 model, and renderings of both these orbit models are included in Figure 4. It is clear that the KI visibility data are reliable at their stated uncertainties, and the most plausible hypothesis is that the VLBI relative astrometry near periastron contains biases associated with the enhanced flaring activity documented by Massi et al. (2002, 2006). This astrometric bias may be naturally explained in the helmet streamer scheme recently proposed by Massi et al. (2008). However, a more detailed analysis, which we defer to a forthcoming paper, will be necessary to understand better the origin of the apparent offset between the radio sources and the underlying stars.

For our final A-subsystem orbit estimation we conducted a joint orbit modeling with the A-B astrometry and RV data sets presented in the B2012 companion paper. The basis of the joint modeling recognizes that RV motions of the A-component stars also include the motion of the A barycenter-moving under the gravitational influence of the other V773 Tau components and dominated by the A-B orbital motion. B2012 describe the joint

\footnotetext{
11 As will become apparent momentarily, the emission is indeed associated with the two stars, but in some cases, it is not exactly coincident with them. 12 Our methods for orbit modeling with heterogenous RV and astrometric/visibility data sets are described in Boden et al. (2000) and are not repeated here. In all the orbit modeling we have weighted all VLBI astrometry consistently with the weighting derived in B2007, at 0.2 mas per axis $1 \sigma$, except as noted for points near periastron in the "Full Hybrid" model.
} 

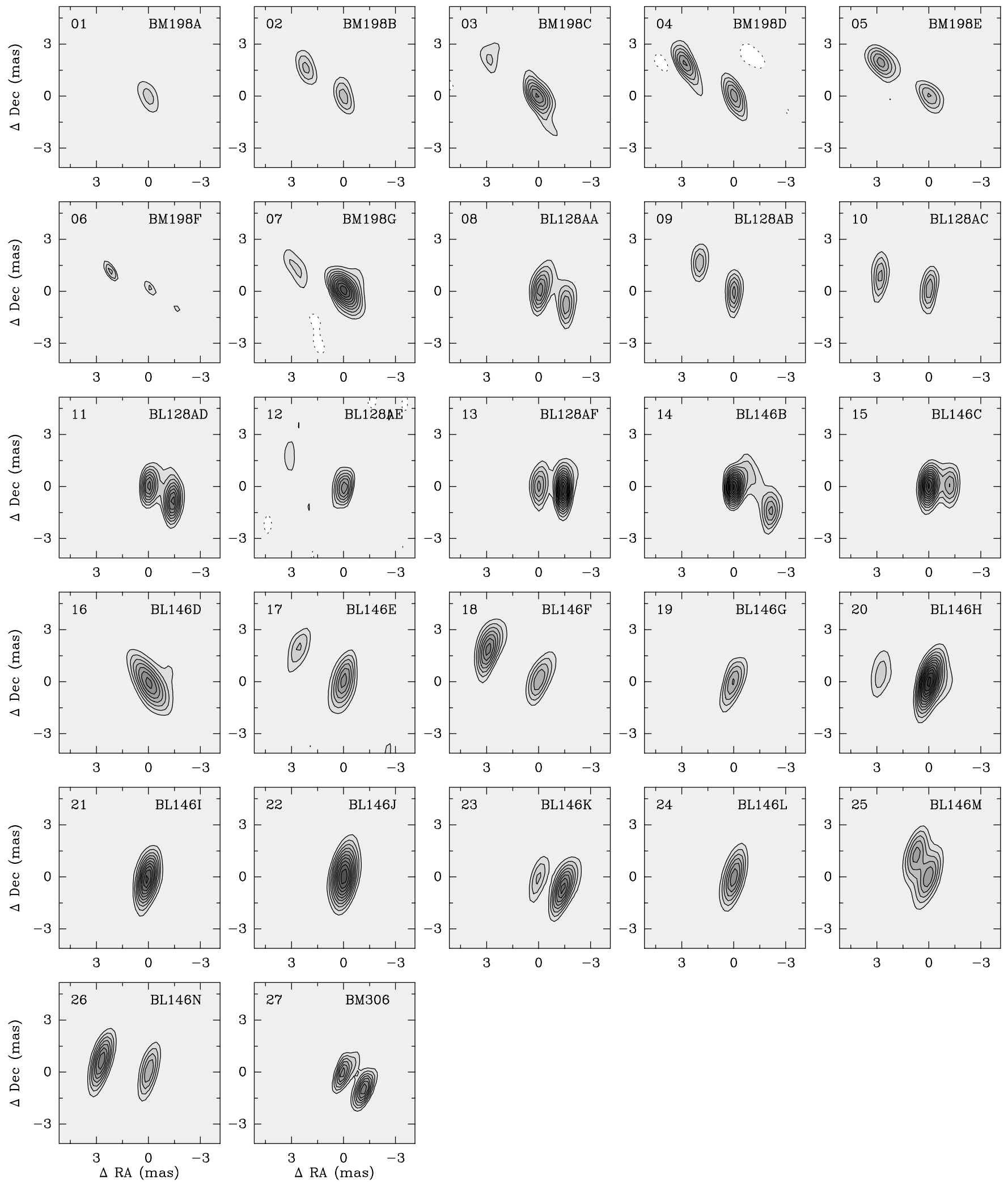

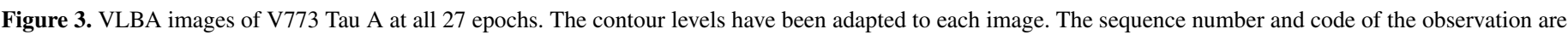

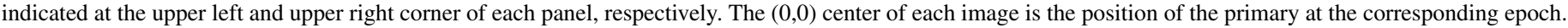

orbit modeling of both the A subsystem and the A-B orbit based on the combined VLBA relative astrometry, KI visibility, highresolution imaging, and expanded RV data sets. Table 4 gives a direct comparison between the orbit models from B2007 and the "Joint Solution" model derived in B2012 based in part on data presented here; in all cases the orbital parameters between the two models (which share a significant amount of underlying data) are in excellent statistical agreement. For all subsequent analysis, we adopt this "Joint Solution" model for the updated V773 Tau A orbit. 
Table 2

Measured Source Positions of the Two Components in V773 Tau, and Orbit Phase $(\phi)$ of the System at All Epochs

\begin{tabular}{|c|c|c|c|c|c|c|c|c|c|}
\hline \multirow[t]{2}{*}{ Epoch } & \multicolumn{4}{|c|}{ Primary (Aa) } & \multicolumn{4}{|c|}{ Secondary $(\mathrm{Ab})$} & \multirow[t]{2}{*}{$\phi$} \\
\hline & $\begin{array}{c}\alpha(\mathrm{J} 2000.0) \\
04^{\mathrm{h}} 14^{\mathrm{m}}\end{array}$ & $\sigma_{\alpha}$ & $\begin{array}{c}\delta(\mathrm{J} 2000.0) \\
28^{\circ} 12^{\prime}\end{array}$ & $\sigma_{\delta}$ & $\begin{array}{c}\alpha(\mathrm{J} 2000.0) \\
04^{\mathrm{h}} 14^{\mathrm{m}}\end{array}$ & $\sigma_{\alpha}$ & $\begin{array}{c}\delta(\mathrm{J} 2000.0) \\
28^{\circ} 12^{\prime}\end{array}$ & $\sigma_{\delta}$ & \\
\hline 01 & $12^{\mathrm{s} .9190149}$ & 0.0000136 & $12^{\prime \prime} .228446$ & $0^{\prime \prime} 000200$ & ND & ND & ND & ND & 0.32 \\
\hline 02 & $12^{s} .9190237$ & $0^{s} .0000026$ & $12^{\prime \prime} .229524$ & 0.000059 & $12^{s .9191887}$ & $0^{\text {s. }} .0000027$ & $12^{\prime \prime} .231170$ & 0.000062 & 0.34 \\
\hline 03 & $12^{\mathrm{s} .9190008}$ & 0.0000036 & $12^{\prime \prime} .229220$ & 0.000077 & $12^{\mathrm{s} .9192084}$ & 0.0000084 & $12^{\prime \prime} .231515$ & 0.000215 & 0.34 \\
\hline 04 & $12^{\mathrm{s}} .9190131$ & 0.0000050 & $12^{\prime \prime} .229236$ & $0^{\prime \prime} 000111$ & $12^{\mathrm{s}} .9192214$ & 0.0000056 & $12^{\prime \prime} .231057$ & 0.000124 & 0.37 \\
\hline 05 & $12^{\mathrm{s} .9189953}$ & 0.0000036 & $12^{\prime \prime} .228788$ & 0.000063 & $12^{\mathrm{s} .9192051}$ & 0.0000036 & $12^{\prime \prime} .230700$ & 0.000058 & 0.39 \\
\hline 06 & $12^{\mathrm{s} .9190069}$ & 0.0000158 & $12^{\prime \prime} .228791$ & 0.000168 & $12^{\mathrm{s} .9192015}$ & 0.0000093 & $12 " .229800$ & 0.000256 & 0.41 \\
\hline 07 & $12^{\mathrm{s} .9190049}$ & 0.0000031 & $12^{\prime \prime} .228361$ & 0.000059 & $12^{\mathrm{s}} .9192194$ & 0.0000077 & $12^{\prime \prime} .229758$ & 0.000161 & 0.43 \\
\hline 08 & $12^{s} .9215399$ & $0^{s} .0000007$ & $12^{\prime \prime} 202276$ & 0.000019 & $12^{s .9214304}$ & $0^{\text {s. }} .0000007$ & $12^{\prime \prime} .201418$ & 0.000024 & 0.99 \\
\hline 09 & $12^{\mathrm{s}} .9211595$ & 0.0000011 & 12 ." 196132 & 0.000037 & $12^{\mathrm{s}} .9213080$ & 0.0000015 & 12 .' 197895 & 0.000042 & 0.32 \\
\hline 10 & $12^{\mathrm{s}} .9207461$ & 0.0000017 & $12^{\prime \prime} 191012$ & 0.000056 & $12^{\mathrm{s}} .9209591$ & 0.0000023 & $12^{\prime \prime} 191822$ & 0.000076 & 0.63 \\
\hline 11 & $12^{\mathrm{s}} .9211497$ & 0.0000007 & $12^{\prime \prime} .186528$ & $0^{\prime \prime} 000020$ & $12^{s} .9210497$ & 0.0000007 & $12^{\prime \prime} .185703$ & 0.000023 & 0.01 \\
\hline 12 & 12.9218068 & $0^{s} .0000033$ & $12^{\prime \prime} 181160$ & 0.000085 & ND & ND & ND & ND & 0.42 \\
\hline 13 & $12^{\mathrm{s} .9226934}$ & 0.0000011 & $12^{\prime \prime} 178983$ & 0.000035 & $12^{s} .9225894$ & 0.0000005 & $12^{\prime \prime} 178760$ & 0.000019 & 0.08 \\
\hline 14 & $12^{\mathrm{s} .9240466}$ & 0.0000007 & $12^{\prime \prime} .156238$ & 0.000015 & $12^{s .9239000}$ & 0.0000018 & $12^{\prime \prime} .155069$ & 0.000045 & 0.97 \\
\hline 15 & $12^{s} .9240254$ & 0.0000004 & 12 ". 155202 & $0^{\prime \prime} 000011$ & $12^{\mathrm{s} .9239336}$ & 0.0000008 & $12^{\prime \prime} 155274$ & 0.000024 & 0.08 \\
\hline 16 & NR & $\mathrm{NR}$ & NR & NR & $\mathrm{NR}$ & NR & NR & NR & 0.22 \\
\hline 17 & $12^{\mathrm{s} .9239237}$ & 0.0000043 & $12^{\prime \prime} .153290$ & $0^{\prime \prime} 000120$ & $12^{s} .9241176$ & 0.0000068 & $12^{\prime \prime} 155149$ & 0.'000176 & 0.34 \\
\hline 18 & $12^{s} .9238939$ & $0^{\mathrm{s} .0000018}$ & $12^{\prime \prime} 153463$ & 0.000042 & $12^{\mathrm{s}} .9241178$ & 0.0000012 & $12^{\prime \prime} 155184$ & 0.000032 & 0.43 \\
\hline 19 & $12^{\mathrm{s}} .9238880$ & $0^{s} .0000036$ & $12^{\prime \prime} 153258$ & 0.000097 & ND & ND & ND & ND & 0.53 \\
\hline 20 & $12^{\mathrm{s} .9239130}$ & $0^{\mathrm{s}} .0000010$ & $12^{\prime \prime} 152788$ & 0.000027 & $12^{s .9241227}$ & 0.0000044 & $12^{\prime \prime} .153447$ & 0.000112 & 0.65 \\
\hline 21 & NR & NR & NR & NR & NR & NR & NR & NR & 0.77 \\
\hline 22 & NR & NR & NR & NR & NR & NR & NR & NR & 0.88 \\
\hline 23 & $12^{s} .9240573$ & $0^{\mathrm{s}} .0000011$ & $12^{\prime \prime} 152076$ & 0.000036 & $12^{s} .9239482$ & $0^{\mathrm{s}} .0000006$ & $12^{\prime \prime} 151553$ & 0.000016 & 0.04 \\
\hline 24 & NR & NR & NR & NR & NR & NR & NR & NR & 0.16 \\
\hline 25 & $12^{\mathrm{s} .9239225}$ & $0^{\mathrm{s} .0000012}$ & $12^{\prime \prime} 150350$ & 0.000034 & $12^{\mathrm{s}} .9239774$ & 0.0000010 & $12^{\prime \prime} 151708$ & 0.000029 & 0.24 \\
\hline 26 & $12^{\mathrm{s}} .9237209$ & 0.0000006 & $12^{\prime \prime} .149354$ & 0.000022 & $12^{\mathrm{s} .9239280}$ & 0.0000005 & 12 ". 149924 & $0.0^{\prime} 000014$ & 0.64 \\
\hline 27 & 12.9272872 & $0^{s} .0000012$ & 12 ". 100333 & 0.000026 & $12^{\mathrm{s} .9272011}$ & 0.0000009 & $12^{\prime \prime} 099345$ & 0.000021 & 0.95 \\
\hline
\end{tabular}

Notes. NR: not resolved; ND: not detected.

Table 3

Measured Separations and Computed Barycenter Positions of the System

\begin{tabular}{|c|c|c|c|c|c|c|c|c|}
\hline Epoch & $\begin{array}{c}\Delta \alpha \\
\text { (mas) }\end{array}$ & $\sigma_{\Delta \alpha}$ & $\begin{array}{c}\Delta \delta \\
\text { (mas) }\end{array}$ & $\sigma_{\Delta \delta}$ & $\begin{array}{c}\alpha(\mathrm{J} 2000.0) \\
04^{\mathrm{h}} 14^{\mathrm{m}}\end{array}$ & $\sigma_{\alpha}$ & $\begin{array}{c}\delta(\mathrm{J} 2000.0) \\
28^{\circ} 12^{\prime}\end{array}$ & $\sigma_{\delta}$ \\
\hline 02 & 2.18 & 0.04 & 1.65 & 0.06 & 12.9190987 & 0.0000019 & $12^{\prime \prime} .230272$ & 0.'000043 \\
\hline 03 & 2.74 & 0.11 & 2.29 & 0.22 & $12^{\mathrm{s} .9190952}$ & $0^{\mathrm{s}} .0000043$ & $12^{\prime \prime} .230263$ & 0.000106 \\
\hline 04 & 2.75 & 0.08 & 1.82 & 0.13 & 12.9191078 & 0.0000037 & $12^{\prime \prime} .230064$ & 0.000083 \\
\hline 05 & 2.77 & 0.06 & 1.91 & 0.07 & $12^{\text {s.91909 }}$ & 0.0000025 & $12^{\prime \prime} .229657$ & 0.000043 \\
\hline 06 & 2.90 & 0.15 & 3.43 & 0.23 & $12^{\mathrm{s}} .9190954$ & 0.0000096 & $12^{\prime \prime} .229250$ & 0.000148 \\
\hline 07 & 2.83 & 0.11 & 1.40 & 0.16 & 12.9191024 & 0.0000039 & $12^{\prime \prime} 228996$ & 0.000080 \\
\hline 08 & -1.45 & 0.01 & -0.86 & 0.03 & $12^{\mathrm{s} .9214901}$ & 0.0000005 & $12^{\prime \prime} 201886$ & 0.000015 \\
\hline 09 & 1.96 & 0.02 & 1.76 & 0.06 & $12^{\mathrm{s}} .9212270$ & 0.0000009 & $12^{\prime \prime} 196933$ & 0.000028 \\
\hline 10 & 2.82 & 0.04 & 0.81 & 0.09 & 12.9208429 & 0.0000014 & $12^{\prime \prime} 191380$ & 0.000046 \\
\hline 11 & -1.32 & 0.01 & -0.83 & 0.03 & $12^{s .9211042}$ & 0.0000005 & $12^{\prime \prime} .186153$ & 0.000015 \\
\hline 13 & -1.37 & 0.02 & -0.22 & 0.04 & $12^{\mathrm{s}} .9226461$ & 0.0000006 & $12^{\prime \prime} 178882$ & 0.000021 \\
\hline 14 & -1.94 & 0.02 & -1.17 & 0.05 & $12^{\mathrm{s}} .9239800$ & 0.0000009 & $12^{\prime \prime} .155707$ & 0.000022 \\
\hline 15 & -1.21 & 0.01 & 0.07 & 0.03 & $12^{\mathrm{s}} .9239837$ & 0.0000004 & $12^{\prime \prime} .155235$ & 0.000013 \\
\hline 17 & 2.56 & 0.11 & 1.86 & 0.21 & $12^{\mathrm{s} .9240118}$ & $0^{\mathrm{s}} .0000038$ & $12^{\prime \prime} .154135$ & 0.000103 \\
\hline 18 & 2.96 & 0.03 & 1.72 & 0.05 & $12^{\mathrm{s} .9239957}$ & 0.0000011 & $12^{\prime \prime} .154245$ & 0.000027 \\
\hline 20 & 2.77 & 0.06 & 0.66 & 0.12 & $12^{s .9240083}$ & 0.0000021 & $12^{\prime \prime} .153088$ & 0.000053 \\
\hline 23 & -1.44 & 0.02 & -0.52 & 0.04 & $12^{s} .9240077$ & 0.0000007 & $12^{\prime \prime} 151838$ & 0.000021 \\
\hline 25 & 3.37 & 0.02 & 2.05 & 0.04 & 12.9239475 & 0.0000008 & $12^{\prime \prime} 150967$ & 0.000023 \\
\hline 26 & 2.74 & 0.01 & 0.57 & 0.03 & $12^{\mathrm{s}} .9238150$ & $0^{\mathrm{s}} .0000004$ & $12^{\prime \prime} 149613$ & $0^{\prime \prime} 000014$ \\
\hline 27 & -1.14 & 0.02 & -0.99 & 0.03 & $12^{\mathrm{s}} .9272481$ & 0.0000008 & $12^{\prime \prime} 099884$ & 0.000017 \\
\hline
\end{tabular}

Given the agreement between the "Joint Solution" model and the B2007 orbit model, the updated physical parameters are highly consistent with B2007 estimates. Component dynamical masses resulting from this orbit model are $1.59 \pm 0.12 M_{\odot}$ and $1.323 \pm 0.079 M_{\odot}$ for the primary and the secondary, respectively-consistent with the B2007 estimates to 3\% and $0.4 \sigma$. Similarly, the system distance estimate derived from the updated orbit model is $135.7 \pm 3.2 \mathrm{pc}$, again highly consistent with the value from B2007. As a final note, we should stress that the present model must still be considered preliminary 


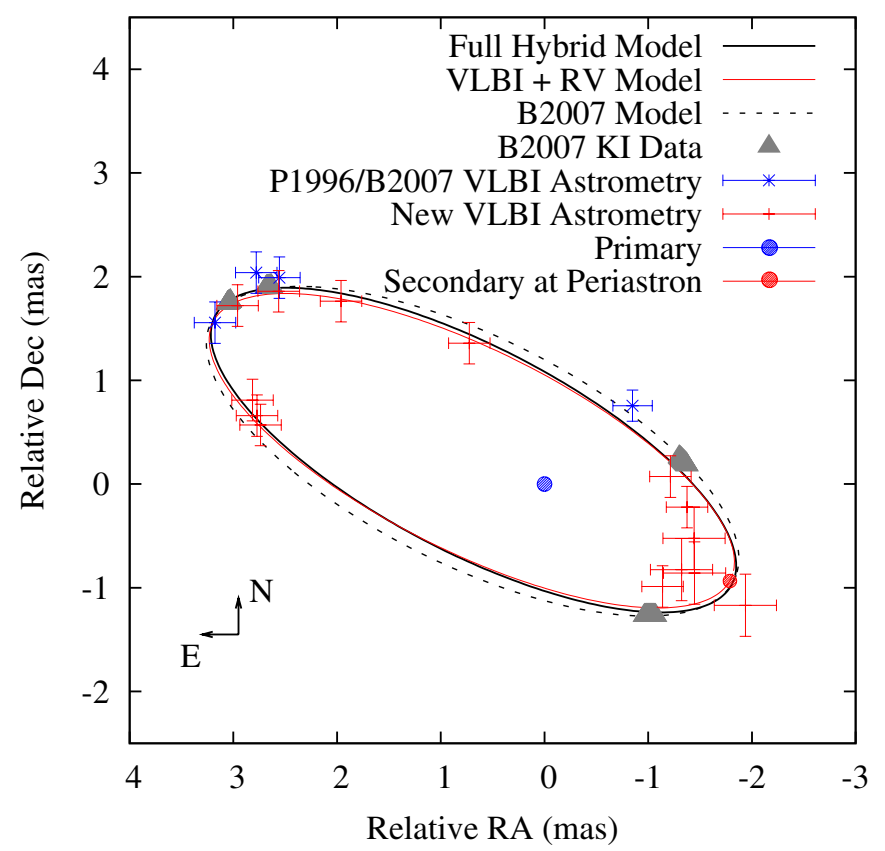

Figure 4. VLBI relative astrometry and orbit models of V773 Tau A. Here VLBI relative astrometry from P1996, B2007, and this work is shown with orbit model renderings for the B2007 orbit and two models derived here (the "VLBI + RV" and "Full Hybrid" models described in the text). The primary (Aa) component is shown at the origin and the secondary $(\mathrm{Ab})$ at periastron. The apparent sizes of the V773 Tau A components are estimated by SED modeling (B2007) and rendered to scale.

Table 4

V773 Tau A Orbital Parameters

\begin{tabular}{lcc}
\hline \hline Orbital Parameter & B2007 & "Joint Solution" \\
\hline Period (days) & $51.1039 \pm 0.0021$ & $51.1003 \pm 0.0022$ \\
$T_{o}(\mathrm{MJD})$ & $53059.73 \pm 0.33$ & $53059.92 \pm 0.33$ \\
$e$ & $0.2717 \pm 0.0085$ & $0.2710 \pm 0.0072$ \\
$K_{A}\left(\mathrm{~km} \mathrm{~s}^{-1}\right)$ & $35.90 \pm 0.53$ & $35.74 \pm 0.66$ \\
$K_{B}\left(\mathrm{~km} \mathrm{~s}^{-1}\right)$ & $41.5 \pm 1.4$ & $43.0 \pm 1.4$ \\
$\gamma\left(\mathrm{km} \mathrm{s}^{-1}\right)$ & $0.02 \pm 0.32$ & $0.0 \pm 0.32$ \\
$\omega_{A}(\mathrm{deg})$ & $4.6 \pm 2.4$ & $5.2 \pm 2.7$ \\
$\Omega(\mathrm{deg})$ & $63.5 \pm 1.7$ & $63.3 \pm 1.1$ \\
$i(\mathrm{deg})$ & $66.0 \pm 2.4$ & $67.6 \pm 1.5$ \\
$a(\mathrm{mas})$ & $2.811 \pm 0.047$ & $2.837 \pm 0.035$ \\
\hline
\end{tabular}

as astrometric and $\mathrm{RV}$ observations to assess the gravitational effect of the two other members of the system (V773 Tau B and $\mathrm{C}$, reported in B2012) are ongoing.

\subsection{Absolute Astrometry and Parallax Determination}

In addition to their separation, the VLBA provides the absolute position of each of the radio sources (Table 2). ${ }^{13}$ Once the orbital motion of the system has been determined, it can be subtracted from the measured positions to obtain the coordinates of the barycenter of the system. If the measured separation between the primary and the secondary is $\mathbf{r}$, then the separation $\mathbf{r}_{1}$ between the primary to the barycenter is given by

$$
\mathbf{r}_{1}=\frac{m_{s}}{m_{s}+m_{p}} \mathbf{r},
$$

\footnotetext{
${ }^{13}$ Strictly, the VLBA provides the angular offset between the source and the main calibrator. However, that calibrator is a distant quasar which can be assumed to be fixed, and whose coordinates are measured relative to the International Celestial Reference Frame. Thus, the positions delivered by the VLBA are very nearly absolute.
}

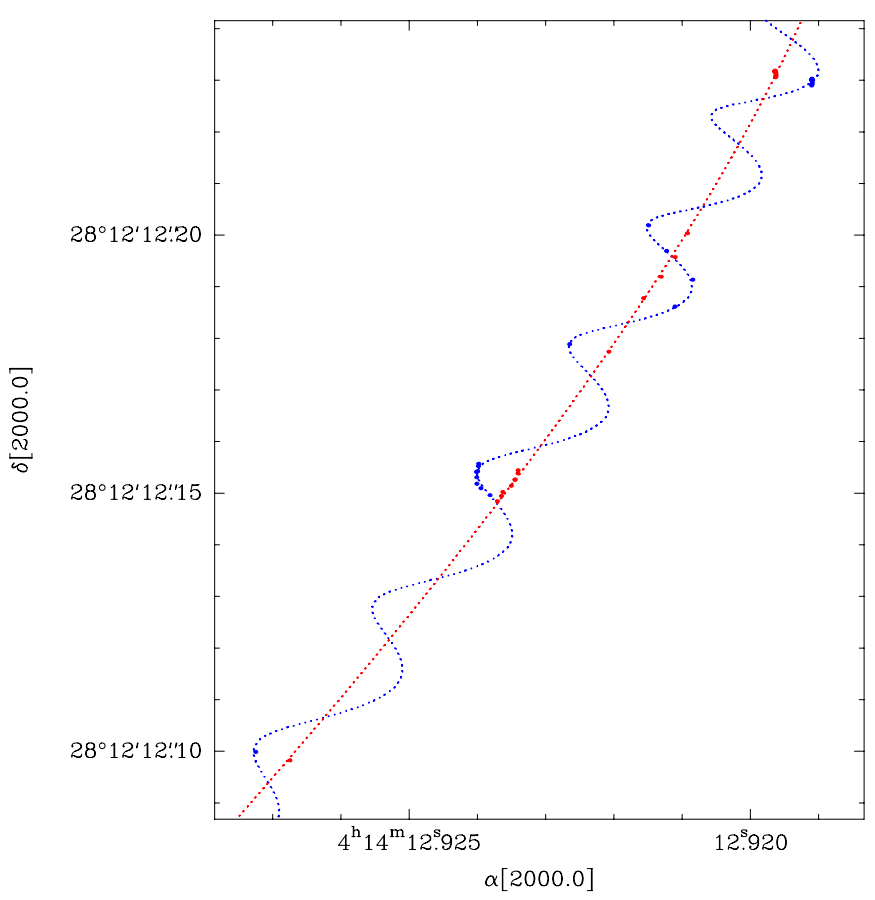

Figure 5. Measured barycenter positions (blue symbols) and best astrometric fit (blue dotted curve) for V773 Tau A. The observed positions are shown as ellipses, whose sizes are the error bars. The red symbols and curve show the same positions but with the parallactic contribution removed. Note the clear curvature of the trajectory of the barycenter of V773 Tau A.

where $m_{p}$ and $m_{s}$ are the masses of the primary and secondary, respectively, deduced from the orbital fit. Clearly, this strategy can only be applied directly to our 20 observations where the system is found to be double. We did not attempt to correct for the displacement of the radio emission from the stars themselves near periastron since it is not clear whether this effect preferentially affects one specific star or both equally.

A fit to the resulting 20 barycenter positions (given explicitly in Table 3) with a combination of a trigonometric parallax and a uniform proper motion provides a very poor agreement with the observations. This is not particularly surprising since V773 Tau A will be affected by the gravitational influence of the B component on the timescale of our data set. In particular, recent infrared observations (B2012) have shown that the mass of component $\mathrm{B}$ is $2.35 \pm 0.67 M_{\odot}$ (i.e., comparable to that of component $\mathrm{A}$ ), while the semimajor axis and period of the $\mathrm{A}-\mathrm{B}$ orbit are approximately $15 \mathrm{AU}$ and $26 \mathrm{yr}$, respectively. Thus, our observations cover a time span corresponding to about a fifth of the total orbital period, and the acceleration of the barycenter of V773 Tau A is expected to be significant. Indeed, a fit with a uniformly accelerated proper motion provides an excellent description of the data (Figure 5). The astrometric elements corresponding to that fit are

$$
\begin{aligned}
\alpha_{\mathrm{J} 2006.97} & =04^{\mathrm{h}} 14^{\mathrm{m}} 12^{\mathrm{s}} .922447 \pm 0.000010 \\
\delta_{\mathrm{J} 2006.97} & =28^{\circ} 12^{\prime} 12^{\prime \prime} 170682 \pm 0.000097 \\
\mu_{\alpha} \cos \delta_{\mathrm{J} 2006.97} & =17.092 \pm 0.077 \mathrm{mas}^{-1} \\
\mu_{\delta \mathrm{J} 2006.97} & =-24.030 \pm 0.053 \mathrm{mas} \mathrm{yr}^{-1} \\
a_{\alpha} \cos \delta & =2.60 \pm 0.60 \mathrm{mas} \mathrm{yr}^{-2} \\
a_{\delta} & =-1.51 \pm 0.52 \mathrm{mas} \mathrm{yr}^{-2} \\
\pi & =7.70 \pm 0.19 \text { mas. }
\end{aligned}
$$


The reference epoch (2006.97) corresponds to the median epoch of the observations used in the astrometric fit. The post-fit rms is 0.34 mas in right ascension and 0.29 mas in declination, in good agreement with our assumption that the errors on the separation between the sources are of the order of 0.2-0.3 mas per axis (see Section 3.1). The acceleration found here is in excellent agreement with the expected acceleration of the barycenter of V773 Tau A due to the gravitational pull of component V773 Tau B. In the companion paper, B2012 report on multi-epoch near-infrared observations of and orbit modeling for the A-B subsystem. The mean A-barycenter acceleration estimated from that orbit model during the time span covered by our VLBA observations is $a_{\alpha} \cos \delta=2.21 \pm$

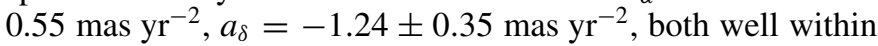
$1 \sigma$ of the VLBA determination. It is important to stress that the two determinations of the acceleration are entirely independent-one is based on absolute astrometry of radio observations, while the other rests on relative astrometry of near-infrared data. Thus, the concordance of the two results lends strong support to both analyses.

The distance corresponding to the trigonometric parallax obtained from our fit is $129.9 \pm 3.2 \mathrm{pc}$. This is in agreement with the-almost entirely independent-distance obtained from the orbit model at the $4.5 \%$, or $1 \sigma$ level. ${ }^{14}$ This very good level of agreement validates both methods and demonstrates that the quoted errors represent the true accuracy (and not just the precision) of the results. By taking the mean of the two values, we obtain our favored estimate of the distance to V773 Tau A: $d=132.8 \pm 2.3$ pc.

Our distance determination is only very marginally consistent with the result $\left(d=148.4_{-5.3}^{+5.7} \mathrm{pc}\right)$ obtained by Lestrade et al. (1999) using multi-epoch VLBI observations similar to those presented here. The trustworthiness of our astrometry is demonstrated by the concordance of our parallax, acceleration, and proper motion (see below) with those of entirely independent infrared observations. Lestrade et al. (1999), on the other hand, did not take into account the binarity of the A subsystem or the effect of the B component in their astrometric modeling. As a consequence, the mathematical function used in their fit did not provide an adequate description of the modeled data, and the quoted errors on the fitted parameters do not properly reflect actual uncertainties. To reconcile the results of Lestrade et al. (1999) with the conclusions of our astrometric analyses, the errors quoted by Lestrade et al. (1999) must be multiplied by a factor of about three. We conclude that the present work improves the actual uncertainty on the distance to V773 Tau by nearly one order of magnitude from about 15-20 pc down to 2.4 pc over the results of Lestrade et al. (1999).

Another apparent discrepancy with the results of Lestrade et al. (1999) must be briefly mentioned here. Lestrade et al. (1999) reported proper motions for V773 Tau A of $\mu_{\alpha} \cos \delta=$ $0.42 \pm 0.29$ mas $\mathrm{yr}^{-1}, \mu_{\delta}=-23.25 \pm 0.25 \mathrm{mas}_{\mathrm{yr}}^{-1}$. These figures are similar to those reported in the Hipparcos

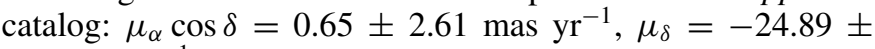
$1.81 \mathrm{mas} \mathrm{yr}^{-1}$ (Perryman et al. 1997), but are very different from those found here especially in right ascension. The difference is $\Delta\left(\mu_{\alpha} \cos \delta\right)=+16.7 \pm 0.3$ mas $\mathrm{yr}^{-1}, \Delta\left(\mu_{\delta}\right)=-0.8 \pm$

\footnotetext{
14 Here, and in the rest of the paper, we test the consistency of results by examining the number of sigmas required to obtain overlapping error bars. In the present case, the $1 \sigma$ error bar on the orbit distance estimate is $132.5 \mathrm{pc}<d<138.9 \mathrm{pc}$, while for the trigonometric parallax distance, it is $126.7 \mathrm{pc}<d<133.1 \mathrm{pc})$. Since they overlap, we consider the results consistent within $1 \sigma$.
}

0.3 mas $\mathrm{yr}^{-1}$. The difference once again reflects the nonuniformity of the barycentric motion of V773 Tau A due to the gravitational effect of V773 Tau B. While the mean epochs of the observations reported by Lestrade et al. (1999) and of the Hipparcos satellite are very similar (1993.88 and 1991.25, respectively), our observations are significantly more recent (2006.97). According to the A-B orbit published in B2012, the instantaneous proper motion of the A barycenter of V773 Tau (measured relative to the barycenter of the entire

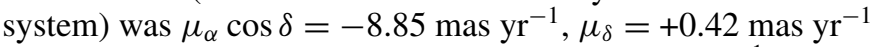

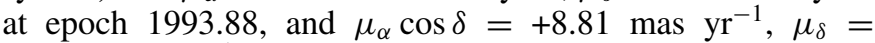

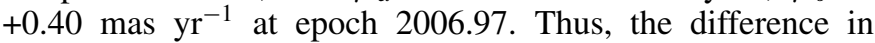
proper motion between 1993.88 and 2006.97 according to the A-B orbital fit is $\Delta\left(\mu_{\alpha} \cos \delta\right)=+17.66$ mas $\mathrm{yr}^{-1}, \Delta\left(\mu_{\delta}\right)=$ $-0.02 \mathrm{mas} \mathrm{yr}^{-1}$, in very good agreement with the measured values. Indeed, knowing the relative expected proper motion between the barycenter of V773 Tau A and the barycenter of V773 Tau as a whole, we can estimate the proper motion of the barycenter of V773 Tau as a whole. We obtain $\mu_{\alpha} \cos \delta=+8.3 \pm$

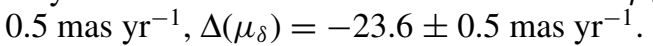

We mentioned earlier that the source was single in 7 of our 27 observations. With the help of the astrometric fits, it is possible to determine the state of the system at those epochs. In three of the cases (epochs 1, 12, and 19), the single detected source appears to be the primary of the system. In all three cases, the source was weak ( $\sim 1 \mathrm{mJy}$ ) and the system was near apastron. Evidently, the secondary had faded below our detection limit at these epochs. In the remaining four observations (epochs 16, 21, 22, and 24), the single detected source is bright (at least several mJy) and its position is intermediate between the expected positions of the primary and the secondary. In all cases, the system was at an orbit phase of about 0.2 before or after periastron. In this situation, the two stars are located in projection almost exactly north-south of each other, with a projected separation of about 1 mas (see Figure 4). Since our resolution in the north-south direction is about 2 mas, we do not expect to resolve the two stars in this situation, but instead to detect a single source slightly elongated in the north-south direction. This is in fact what happens (see Figure 3). Indeed, the mean deconvolved FWHM size of the emission for these four epochs is 1.33 mas, 50\% larger than the corresponding figure ( 0.89 mas) when the source is double or single but near apastron.

\subsection{The Distance to the L1495 Region of Taurus}

In projection, V773 Tau is located toward the dark cloud Lynds 1495 in the central region of Taurus. Two other young stars with recent VLBA-based parallax measurements (Hubble 4 and HDE 283572; Torres et al. 2007) are located in the same portion of Taurus. Interestingly, they appear to be at very similar distances $(132.8 \pm 0.5 \mathrm{pc}$ for Hubble 4 and $128.5 \pm 0.6 \mathrm{pc}$ for HDE 283572, against $132.8 \pm 2.3 \mathrm{pc}$ for V773 Tau). The proper motions of Hubble 4 and HDE $283572\left(\mu_{\alpha} \cos \delta=\right.$

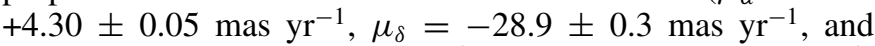
$\mu_{\alpha} \cos \delta=+8.88 \pm 0.06 \operatorname{mas~yr}^{-1}, \mu_{\delta}=-26.6 \pm 0.1$ mas yr $^{-1}$, respectively-Torres et al. 2007) are similar to one another, and to that of the barycenter of V773 Tau $\left(\mu_{\alpha} \cos \delta=+8.3\right.$

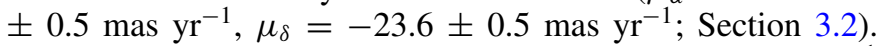
The RVs of the three stars are also similar: $15.0 \pm 1.7 \mathrm{~km} \mathrm{~s}^{-1}$ for Hubble 4 (Hartmann et al. 1986), $15.0 \pm 1.5 \mathrm{~km} \mathrm{~s}^{-1}$ for HDE 283572 (Walter et al. 1988), and $16.38 \pm 0.52 \mathrm{~km} \mathrm{~s}^{-1}$ for V773 Tau (B2012). Finally, the extinction measured toward the V773 Tau system (B2012) is consistent with extinction estimated by Schmalzl et al. (2010) for that portion of L1495. 
Table 5

Source Fluxes and Brightness Temperatures

\begin{tabular}{|c|c|c|c|c|c|c|c|}
\hline \multirow[t]{2}{*}{ Epoch $^{\mathrm{a}}$} & \multicolumn{2}{|c|}{ Primary (Aa) } & \multicolumn{2}{|c|}{ Secondary (Ab) } & \multirow[b]{2}{*}{$\begin{array}{c}f_{v_{A a}}+f_{v_{A b}} \\
(\mathrm{mJy})\end{array}$} & \multirow[b]{2}{*}{$f_{v_{A b}} / f_{v_{A a}}$} & \multirow[b]{2}{*}{$\begin{array}{c}\mathrm{rms} \\
(\mathrm{mJy})\end{array}$} \\
\hline & $\begin{array}{c}f_{v} \\
(\mathrm{mJy})\end{array}$ & $\begin{array}{c}T_{b} \\
\left(10^{6} \mathrm{~K}\right)\end{array}$ & $\begin{array}{c}f_{v}^{\mathrm{b}} \\
(\mathrm{mJy})\end{array}$ & $\begin{array}{c}T_{b} \\
\left(10^{6} \mathrm{~K}\right)\end{array}$ & & & \\
\hline 01 & $0.33 \pm 0.07$ & $3.2 \pm 0.7$ & $<0.22$ & $<2.1$ & $0.33 \pm 0.10$ & $<0.66$ & 0.07 \\
\hline 02 & $1.43 \pm 0.08$ & $14.2 \pm 0.8$ & $1.33 \pm 0.08$ & $13.2 \pm 0.8$ & $2.76 \pm 0.11$ & 0.93 & 0.08 \\
\hline 03 & $0.99 \pm 0.07$ & $9.6 \pm 0.7$ & $0.35 \pm 0.07$ & $3.4 \pm 0.7$ & $1.35 \pm 0.10$ & 0.36 & 0.07 \\
\hline 04 & $0.70 \pm 0.07$ & $5.9 \pm 0.6$ & $0.69 \pm 0.07$ & $5.9 \pm 0.6$ & $1.40 \pm 0.10$ & 0.99 & 0.07 \\
\hline 05 & $1.05 \pm 0.08$ & $11.1 \pm 0.8$ & $1.23 \pm 0.08$ & $13.0 \pm 0.8$ & $2.27 \pm 0.11$ & 1.17 & 0.08 \\
\hline 06 & $0.50 \pm 0.08$ & $4.9 \pm 0.8$ & $0.34 \pm 0.08$ & $3.4 \pm 0.8$ & $0.84 \pm 0.12$ & 0.69 & 0.09 \\
\hline 07 & $1.15 \pm 0.07$ & $10.6 \pm 0.6$ & $0.36 \pm 0.07$ & $3.3 \pm 0.6$ & $1.51 \pm 0.09$ & 0.31 & 0.07 \\
\hline 08 & $7.65 \pm 0.15$ & $89.5 \pm 1.8$ & $5.96 \pm 0.16$ & $69.7 \pm 1.8$ & $13.61 \pm 0.22$ & 0.78 & 0.15 \\
\hline 09 & $2.56 \pm 0.11$ & $32.0 \pm 1.3$ & $1.99 \pm 0.11$ & $24.9 \pm 1.4$ & $4.55 \pm 0.15$ & 0.79 & 0.11 \\
\hline 10 & $1.77 \pm 0.11$ & $19.7 \pm 1.2$ & $1.49 \pm 0.11$ & $16.6 \pm 1.2$ & $3.25 \pm 0.15$ & 0.84 & 0.10 \\
\hline 11 & $8.66 \pm 0.22$ & $110.1 \pm 2.9$ & $9.18 \pm 0.22$ & $116.7 \pm 2.8$ & $17.84 \pm 0.31$ & 1.06 & 0.21 \\
\hline 12 & $0.80 \pm 0.09$ & $9.7 \pm 1.1$ & $<0.28$ & $<3.4$ & $0.80 \pm 0.13$ & $<0.35$ & 0.09 \\
\hline 13 & $3.23 \pm 0.13$ & $40.8 \pm 1.6$ & $6.23 \pm 0.13$ & $78.7 \pm 1.6$ & $9.46 \pm 0.18$ & 1.93 & 0.12 \\
\hline 14 & $9.56 \pm 0.19$ & $114.7 \pm 2.3$ & $4.13 \pm 0.19$ & $49.5 \pm 2.2$ & $13.69 \pm 0.27$ & 0.43 & 0.20 \\
\hline 15 & $9.23 \pm 0.13$ & $106.2 \pm 1.5$ & $4.51 \pm 0.13$ & $51.8 \pm 1.4$ & $13.74 \pm 0.18$ & 0.49 & 0.12 \\
\hline 16 & $\cdots$ & $\cdots$ & $\cdots$ & $\cdots$ & $3.98 \pm 0.12$ & $\cdots$ & 0.12 \\
\hline 17 & $1.09 \pm 0.12$ & $8.3 \pm 0.9$ & $0.66 \pm 0.12$ & $5.0 \pm 0.9$ & $1.75 \pm 0.16$ & 0.60 & 0.12 \\
\hline 18 & $3.01 \pm 0.13$ & $21.2 \pm 0.9$ & $4.19 \pm 0.13$ & $29.5 \pm 0.9$ & $7.20 \pm 0.18$ & 1.39 & 0.12 \\
\hline 19 & $1.27 \pm 0.12$ & $10.7 \pm 1.0$ & $<0.36$ & $<3.0$ & $1.27 \pm 0.17$ & $<0.28$ & 0.12 \\
\hline 20 & $5.31 \pm 0.13$ & $40.1 \pm 1.0$ & $1.37 \pm 0.13$ & $10.4 \pm 0.9$ & $6.68 \pm 0.18$ & 0.26 & 0.13 \\
\hline 21 & $\cdots$ & $\cdots$ & $\cdots$ & $\cdots$ & $5.63 \pm 0.14$ & $\cdots$ & 0.14 \\
\hline 22 & $\cdots$ & $\cdots$ & $\cdots$ & $\cdots$ & $24.01 \pm 0.20$ & $\cdots$ & 0.21 \\
\hline 23 & $10.66 \pm 0.35$ & $89.1 \pm 2.9$ & $22.68 \pm 0.34$ & $189.8 \pm 2.9$ & $33.34 \pm 0.49$ & 2.13 & 0.34 \\
\hline 24 & $\cdots$ & $\cdots$ & $\cdots$ & $\cdots$ & $15.32 \pm 0.18$ & $\cdots$ & 0.19 \\
\hline 25 & $5.06 \pm 0.13$ & $37.5 \pm 1.0$ & $5.37 \pm 0.14$ & $39.8 \pm 1.0$ & $10.43 \pm 0.19$ & 1.06 & 0.14 \\
\hline 26 & $6.00 \pm 0.11$ & $45.0 \pm 0.9$ & $8.95 \pm 0.11$ & $67.2 \pm 0.9$ & $14.95 \pm 0.16$ & 1.49 & 0.11 \\
\hline 27 & $3.66 \pm 0.13$ & $63.7 \pm 2.2$ & $4.42 \pm 0.13$ & $76.8 \pm 2.2$ & $8.08 \pm 0.18$ & 1.21 & 0.13 \\
\hline
\end{tabular}

Notes.

a For epochs 16, 21, 22, and 24, the two sources are blended so only the total flux is given.

b When the source is not detected, the $3 \sigma$ upper limit is given.

Thus, Hubble 4, HDE 283572, and V773 Tau are not only located in the same portion of Taurus, they are also at similar distances and share the same kinematics: they most likely belong to a common spatio-kinematical sub-group within the Taurus complex. We note that in a previous paper of this series (Torres et al. 2007), we reached an opposite conclusion because we used the distance to V773 Tau and proper motions given by Lestrade et al. (1999). The present work shows that the latter distance was likely overestimated by about $15 \mathrm{pc}$, and that the proper motions have to be corrected for the A-B orbit. We argue that the weighted mean of our three parallax measurements provides a good estimate of the mean distance to Lynds 1495, and that their dispersion is a good measure of the depth of the associated stellar population. We conclude that Lynds 1495 can be taken to be at $131.4 \pm 2.4 \mathrm{pc}$.

\section{PROPERTIES OF THE RADIO EMISSION}

\subsection{Source Flux and Variability}

The radio source associated with V773 Tau A is found to be extremely variable from epoch to epoch (Table 5) with a maximum recorded total flux (at epoch 23) one hundred times larger than the minimum recorded total flux (at epoch 1). There is a clear tendency (Figure 6) for the flux to be higher near periastron and lower around apastron, in agreement with the single-dish results of Massi et al. (2002, 2006). The present data demonstrate for the first time that this tendency holds for both sub-components ( $\mathrm{Aa}$ and $\mathrm{Ab}$ ) of the system (Figure 6). The

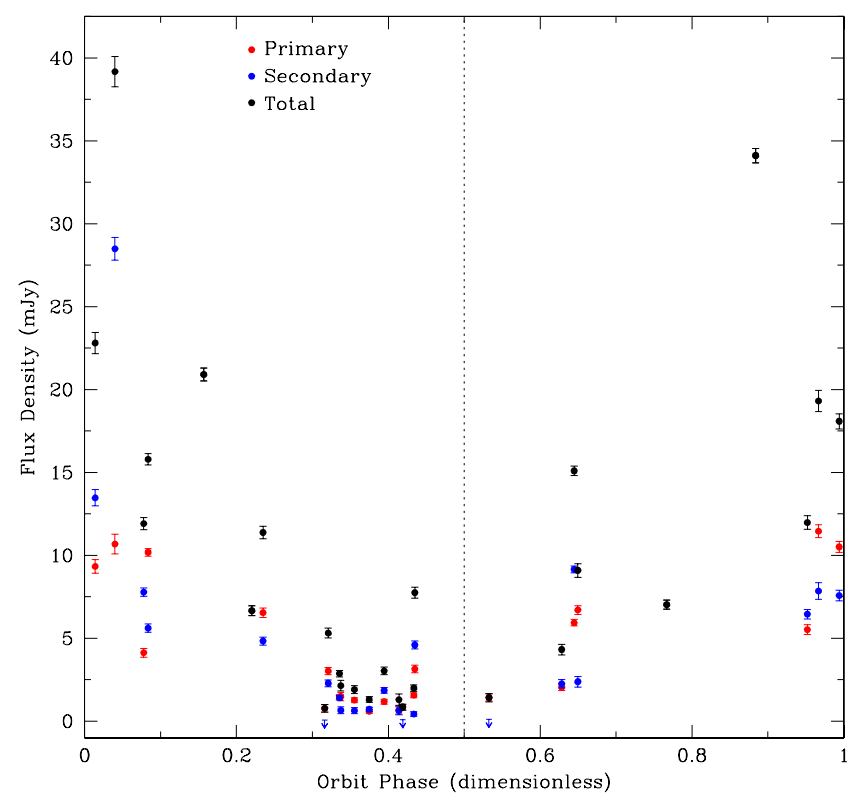

Figure 6. Measured fluxes of V773 Tau as a function of orbit phase ( 0 and 1 mean periastron, whereas 0.5 corresponds to apastron). The total flux of the system as well as the individual fluxes of the sub-components are shown in different colors.

primary is found to be brighter than the secondary in about $65 \%$ of the cases, while the secondary is brighter than the primary in the other $35 \%$. On average, the primary is about $10 \%-15 \%$ 
Table 6

Short-term Variability

\begin{tabular}{lccrr}
\hline \hline Source & $\bar{f}_{v}$ & rms & $f_{v}^{\max }$ & $f_{v}^{\text {min }}$ \\
\cline { 2 - 5 } & \multicolumn{3}{c}{$(\mathrm{mJy})$} \\
\hline $0.3 \leqslant \phi \leqslant 0.7$ (Apastron) & & & \\
V773 Tau A & 5.2 & 4.7 & 14.8 & 0.6 \\
V773 Tau Aa & 2.7 & 2.1 & 5.8 & 0.3 \\
V773 Tau Ab & 2.5 & 3.0 & 9.0 & $<0.1$ \\
$\phi \leqslant 0.2$ or $\phi \geqslant 0.8$ (Periastron) & & & 8.6 \\
V773 Tau A & 20.6 & 9.0 & 40.7 & $<0.2$ \\
V773 Tau Aa & 8.4 & 3.3 & 12.0 & 2.9 \\
V773 Tau Ab & 10.7 & 9.4 & 28.7 & \\
\hline
\end{tabular}

brighter than the secondary. The brightness temperature of both components fluctuates between $3 \times 10^{6} \mathrm{~K}$ and $2 \times 10^{8} \mathrm{~K}$, indicating that the emission is of non-thermal origin.

Short-timescale (intra-epoch) variability is also clearly present in our data. To characterize that variability, we have split each of our observations into $1 \mathrm{hr}$ chunks and measured the flux of each sub-component of the system in those short time intervals. A summary of the results is provided in Table 6, where we consider separately the situation near periastron and around apastron. The mean source flux $\left(\bar{f}_{v}\right.$, measured in $1 \mathrm{hr}$ chunks $)$ is typically four to five times higher near periastron than near apastron, in agreement with our conclusions for epoch-integrated fluxes. The relative variability of the source, as measured by the ratio of the chunk-to-chunk rms to the mean, is found to be between $40 \%$ and $120 \%$ with no significant trend between periastron and apastron.

\subsection{Circular Polarization}

Circular polarization was unambiguously detected at six epochs ${ }^{15}$ (Table 7). The polarization is associated with source $\mathrm{Aa}$ in two cases and with source $\mathrm{Ab}$ in two other cases. The remaining two cases correspond to epochs 22 and 24 when the sources are blended together. The levels of detected circular polarization (between a few and about 10\%) are typical of gyrosynchrotron emission (Dulk 1985). The lack of circular polarization detections in many of our observations presumably indicates that the topology of the magnetic field is complex.

\subsection{Structure and Size of the Emitting Region}

The radio emission detected in our 27 observations is largely confined to two compact sources associated with the two stars in the system. There is only marginal evidence for additional emission in the system-for instance in the form of a faint bridge of emission between the stars at epoch 14 (see Figure 3). Deeper observations of the system should be obtained to confirm the existence of such faint extended emission and analyze its properties in detail. It is clear, in any case, that such emission statistically only represents a small fraction of the total emission.

A compact radio source associated either with component $\mathrm{Aa}$ or with component $\mathrm{Ab}$ is detected a total of 43 times (23 times with source Aa and 20 times with source Ab). Out of this total, the source is found to be completely unresolved 20 times $(46.5 \%)$, and resolved along only one direction in

\footnotetext{
15 It is important to recall that polarization calibration was only possible for the 13 epochs corresponding to project BL146. Thus, our detection rate of circular polarization is roughly $50 \%$.
}

Table 7

Circular Polarization

\begin{tabular}{lcr}
\hline \hline Epoch & Source & $\begin{array}{c}\text { Polarization } \\
(\%)\end{array}$ \\
\hline 15 & $\mathrm{Ab}$ & $10.5 \pm 2.5$ \\
18 & $\mathrm{Aa}$ & $12.3 \pm 3.8$ \\
22 & $\mathrm{Aab}$ & $3.5 \pm 0.3$ \\
23 & $\mathrm{Ab}$ & $1.3 \pm 0.3$ \\
24 & $\mathrm{Aab}$ & $1.8 \pm 0.5$ \\
26 & $\mathrm{Aa}$ & $11.1 \pm 1.3$ \\
\hline
\end{tabular}

another 15 epochs $(35 \%)$. The source is resolved along both directions (and, even then, only marginally) in only eight cases $(18.5 \%)$. The upper limit on the size of the emitting regions along the unresolved dimensions is about 0.7 mas, whereas the deconvolved linear size of the emission along the resolved dimensions is on average 1 mas. Since the radii of both Aa and $\mathrm{Ab}$ are $R_{*} \sim 2 R_{\odot}(\mathrm{B} 2007)$, the radio emission detected here is almost entirely confined to regions of radius $7 R_{*}$, and is often confined to regions of radius smaller than $5 R_{*}$. These figures characterize the size scale of the magnetospheres associated with Aa and Ab.

In summary, the radio emission detected here is confined to very compact (5-7 $R_{*}$ ) regions. It is very variable, occasionally circularly polarized, and has a very high brightness temperature $\left(T_{b} \sim 10^{7} \mathrm{~K}\right)$. All these elements clearly indicate that the emission is primarily of gyrosynchrotron origin (Dulk 1985).

\subsection{A Conundrum}

We showed in Section 4.1 (see Figure 6; Massi et al. 2002, 2006) that the radio flux is strongly dependent on the orbit phase of the binary system, the typical flux near periastron being typically five times higher than the typical flux near apastron. This clearly indicates that the magnetic activity is enhanced when the stars approach one another. Interestingly, however, the separation between the stars at periastron is still about 2 mas or $30 R_{*}$. Thus, even at periastron, the magnetospheres of the two stars (of radii 5-7 $R_{*}$; Section 4.3) do not overlap. How can the radio emission be so strongly dependent on the separation between the stars if the radius of the magnetospheres is several times smaller than the shortest separation between the stars? Even more puzzling, how can the emission still remain almost completely concentrated to 5-7 $R_{*}$ magnetospheres near periastron if the emission is so strongly enhanced by the proximity between the stars?

A possible solution to this conundrum has recently been proposed by Massi et al. (2008) who suggested the existence of two kinds of magnetic structures around the stars in V773 Tau A. The first kind corresponds to compact, closed magnetic loops extending to only a few stellar radii. The second category are semi-open structures similar to the so-called helmet streamers observed around the Sun (e.g., Vourlidas 2006). Those streamers are anchored on the upper edge of a compact closed loop at a few stellar radii above the stellar surface, but they extend to about 30 stellar radii. Reconnections between these extended loops could occur when the stars of V773 Tau A are near periastron, and naturally explain the associated radio flux increase. It is indeed noteworthy that the displacement between the stars and the radio sources observed near periastron (2-3 $R_{*}$; Section 3.1 ) is very similar to the height above the stellar surface at which helmet streamers are expected to be anchored. We note, however, in our observations obtained near periastron that even though the radio 
emission is slightly displaced from the stars, it is still clearly resolved into two compact structures each 5-7 $R_{*}$ across but separated by more than $20 R_{*}$. We find very little evidence for any emission between the stars. In particular, we do not confirm the detection of the mirror magnetic points reported by Massi et al. (2008) in one of their epochs.

\section{CONCLUSIONS}

In this paper, we have described and analyzed new multiepoch VLBA observations of the young stellar system V773 Tau A. The data have been used both to improve the determination of the physical orbit of the compact binary at the center of the system and to measure its trigonometric parallax. We show that the distance to the system obtained from the a priori B2007 and updated orbit model is fully consistent with the direct parallax measurement. The proper motion of the barycenter of V773 Tau A appears to be accelerated, and the measured acceleration vector is in excellent agreement with the expectation based on the inferred gravitational influence of V773 Tau B (B2012). Our results show that V773 Tau is at the same distance and shares the same kinematics as two other nearby young stars (Hubble 4 and HDE 283572) with VLBA parallax measurements. We argue that the mean distance to these three stars $(131.4 \mathrm{pc})$ and their dispersion $(2.4 \mathrm{pc})$ provide a good estimate of the distance and depth of the young population associated with this portion of Taurus (which corresponds to the dark cloud Lynds 1495).

The radio emission detected here is confined to very compact (5-7 $R_{*}$ ) regions associated with the two stars (Aa and $\mathrm{Ab}$ ) in the system. It is very variable, occasionally circularly polarized, and has a very high brightness temperature $\left(T_{b} \sim 10^{7} \mathrm{~K}\right)$. All these elements clearly indicate that the emission is primarily of gyrosynchrotron origin. Each time the system passes near periastron, the radio emission increases by a factor of about five and is slightly displaced from the stars.

R.M.T. and W.H.T.V. acknowledge support by the Deutsche Forschungsgemeinschaft (DFG) through the Emmy Noether Research grant VL 61/3-1. L.L. and L.F.R. acknowledge the financial support of DGAPA, UNAM, and CONACyT, México. L.L. acknowledges financial support from the Guggenheim Foundation and the von Humboldt Stiftung. The National Radio Astronomy Observatory is a facility of the National Science Foundation operated under cooperative agreement by Associated Universities, Inc.

\section{REFERENCES}

Boden, A. F., Creech-Eakman, M. J., \& Queloz, D. 2000, ApJ, 536, 880 Boden, A. F., Torres, G., Duchêne, G., et al. 2012, ApJ, 747, 17 (B2012) Boden, A. F., Torres, G., Sargent, A. I., et al. 2007, ApJ, 670, 1214 (B2007) Briggs, D. S. 1995, BAAS, 27, 1444

Dzib, S., Loinard, L., Mioduszewski, A. J., et al. 2010, ApJ, 718, 610

Dzib, S., Loinard, L., Rodríguez, L. F., Mioduszewski, A. J., \& Torres, R. M. 2011, ApJ, 733, 71

Duchêne, G., Ghez, A. M., McCabe, C., \& Weinberger, A. J. 2003, ApJ, 592, 288 (D2003)

Dulk, G. A. 1985, A\&A, 23, 169

Feigelson, E. D., Welty, A. D., Imhoff, C., et al. 1994, ApJ, 432, 373

Ghez, A. M., Neugebauer, G., \& Matthews, K. 1993, AJ, 106, 2005

Greisen, E. W. 2003, in Information Handling in Astronomy-Historical Vistas, ed. A. Heck (Dordrecht: Kluwer), 109

Hartmann, L., Hewett, R., Stahler, S., \& Mathieu, R. D. 1986, ApJ, 309, 275

Hillenbrand, L. A., \& White, R. J. 2004, ApJ, 604, 741

Kutner, M. L., Rydgren, A. E., \& Vrba, F. J. 1986, AJ, 92, 895

Leinert, Ch., Zinnecker, H., Weitzel, N., et al. 1993, A\&A, 278, 129

Lestrade, J.-F., Preston, R. A., Jones, D. L., et al. 1999, A\&A, 344, 1014

Loinard, L., Mioduszewski, A. J., Rodríguez, L. F., et al. 2005, ApJ, 619, L179

Loinard, L., Torres, R. M., Mioduszewski, A. J., et al. 2007, ApJ, 671, 546

Loinard, L., Torres, R. M., Mioduszewski, A. J., \& Rodríguez, L. F. 2008, ApJ, 675, L29

Martin, E. L., Rebolo, R., Magazzu, A., \& Pavlenko, Ya. V. 1994, A\&A, 282, 503

Mason, B. D., Wycoff, G. L., Hartkopf, W. I., Douglass, G. G., \& Worley, C. E. 2001, AJ, 122, 3466

Massi, M., Forbrich, J., Menten, K. M., et al. 2006, A\&A, 453, 959

Massi, M., Menten, K., \& Neidhöfer, J. 2002, A\&A, 382, 152

Massi, M., Ros, E., Menten, K. M., et al. 2008, A\&A, 480, 489

Mathieu, R. D., Baraffe, I., Simon, M., Stassun, K. G., \& White, R. 2007, in Protostars and Planets V, ed. B. Reipurth, D. Jewitt, \& K. Keil (Tucson, AZ: Univ. Arizona Press), 411

O’Neal, D., Feigelson, E. D., Mathieu, R. D., \& Myers, P. C. 1990, AJ, 100, 1610

Perryman, M. A. C., Lindegren, L., Kovalevsky, J., et al. 1997, A\&A, 323, L49 Phillips, R. B., Lonsdale, C. J., Feigelson, E. D., \& Deeney, B. D. 1996, AJ, 111, 918 (P1996)

Rydgren, A. E., Strom, S. E., \& Strom, K. M. 1976, ApJS, 30, 307

Schmalzl, M., Kainulainen, J., Quanz, S. P., et al. 2010, ApJ, 725, 1327

Torres, R. M., Loinard, L., Mioduszewski, A. J., \& Rodríguez, L. F. 2007, ApJ, 671,1813

Torres, R. M., Loinard, L., Mioduszewski, A. J., \& Rodríguez, L. F. 2009, ApJ, 698,242

Torres, R. M. 2010, PhD thesis, Univ. Nacional Autónoma de México

Vourlidas, A. 2006, in Solar Activity and Its Magnetic Origin, ed. V. Bothmer \& A. Hady (Cambridge: Cambridge Univ. Press), 197

Walter, F. M., Brown, A., Mathieu, R. D., Myers, P. C., \& Vrba, F. J. 1988, AJ, 96, 297

Welty, A. D. 1995, AJ, 110, 776

White, R. J., \& Ghez, A. M. 2001, ApJ, 556, 265

Woitas, J. 2003, A\&A, 406, 685 